\title{
ArcheoSciences
}

Revue d'archéométrie

41-2 | 2017

Varia

\section{Datation dendrochronologique de chalets d'alpage dans la zone cœur du Parc National de la Vanoise}

Tree-ring dating of mountain pasture buildings in the core area of the Vanoise National Park

Melaine Le Roy, Laurent Astrade, Jean-Louis Édouard, Marie-Pierre Bazan et Cristina lancovescu

\section{(2) OpenEdition}

\section{Journals}

Édition électronique

URL : http://journals.openedition.org/archeosciences/4944

DOI : $10.4000 /$ archeosciences. 4944

ISBN : 978-2-7535-7393-2

ISSN : 2104-3728

Éditeur

Presses universitaires de Rennes

Édition imprimée

Date de publication : 8 décembre 2017

Pagination : 7-22

ISBN : 978-2-7535-7391-8

ISSN : 1960-1360

\section{Référence électronique}

Melaine Le Roy, Laurent Astrade, Jean-Louis Édouard, Marie-Pierre Bazan et Cristina lancovescu, «Datation dendrochronologique de chalets d'alpage dans la zone cœur du Parc National de la Vanoise », ArcheoSciences [En ligne], 41-2 | 2017, mis en ligne le 08 décembre 2019, consulté le 01 mai 2019. URL : http://journals.openedition.org/archeosciences/4944 ; DOI : 10.4000/ archeosciences.4944 


\title{
Datation dendrochronologique de chalets d'alpage dans la zone cœur du Parc National de la Vanoise
}

\author{
Tree-ring dating of Mountain Pasture Buildings in the Core Area \\ of the Vanoise National Park
}

\author{
Melaine Le RoY ${ }^{\mathrm{a}}$, Laurent Astrade ${ }^{\mathrm{a}}$, Jean-Louis ÉdOUARD ${ }^{\mathrm{b}}$, \\ Marie-Pierre BAZAN ${ }^{c}$ et Cristina IANCOVESCU ${ }^{\mathrm{d}}$
}

\begin{abstract}
Résumé : Le bâti traditionnel d'altitude a été relativement peu étudié dans les Alpes françaises du Nord. Dans cette étude, cinq bâtiments d'alpage situés dans la zone cour du Parc National de la Vanoise ont fait l'objet d'analyses dendrochronologiques détaillées. L'objectif est d'apporter un premier éclairage sur la temporalité de l'occupation de ces sites et sur les modalités d'utilisation du bois dans l'architecture vernaculaire de ces hautes vallées. Les résultats mettent en évidence plusieurs phases de construction et/ou réparation sur la majorité des sites avec le remploi de nombreuses pièces. La première date d'abattage a été déterminée au milieu du XIv ${ }^{e}$ siècle, mais la première période d'abattage commune à plusieurs bâtiments intervient dans la première moitié du $\mathrm{Xv}^{\mathrm{e}}$ siècle. L'approche dendrochronologique demanderait à être étendue et combinée avec d'autres disciplines (archéologie du bâti, données historiques) afin de mettre en perspective ces données chronologiques. Il existe en outre un besoin majeur d'interventions préventives pour documenter ce patrimoine unique avant rénovation ou ruine totale des bâtiments, conjointement à un travail d'information et de sensibilisation à destination des propriétaires.
\end{abstract}

\begin{abstract}
High-elevation traditional building has been little studied in the northern French Alps. In this study, five mountain pasture buildings located in the core area of the Vanoise National Park have been thoroughly sampled for dendrochronological analyses. The aim is to provide a first insight into the temporality of the occupation of these sites and to characterize the use of wood in vernacular architecture of these high valleys. The results highlight several construction and/or repair-phases at most sites with reuse of many wood pieces. The first felling date was determined in the mid-14 $4^{\text {th }}$ century, but the first felling period concerning several buildings is the first half of the $15^{\text {th }}$ century. The dendrochronological approach would require to be more widely used in the massif and combined with other disciplines (building archaeology, historical data) to put into perspective chronological data presented here. There is also an urgent need for preventive interventions to document this unique heritage before renovation or complete ruin of the buildings, together with an information work and outreach to owners.
\end{abstract}

Mots clé : Alpes du Nord, Alpage, Architecture vernaculaire, Patrimoine bâti, Dendrochronologie.

Keywords: Northern French Alps, Mountain Pasture, Vernacular architecture, Building Heritage, Dendrochronology.

\footnotetext{
a Université Savoie-Mont-Blanc, CNRS, Laboratoire Edytem UMR 5204, Campus scientifique, Pôle Montagne, 73376 LE BOURGET-DU-LAC cedex. (melaine.le-roy@univ-smb.fr), (laurent.astrade@univ-smb.fr)

${ }^{b}$ Aix-Marseille Université, CNRS, Ministère de la Culture et de la Communication, CCJ UMR 7299, 13094 AIX-EN-PROVENCE, France, (jl.edouard@ laposte.net)

"Parc National de la Vanoise, Immeuble "Le France ", 53 avenue de Haute-Tarentaise, 73700 BourG-SAINT-MAURICE. (mpbazan@yahoo.fr)

'Architecte libéral. (arch.ci68@gmail.com)
} 


\section{INTRODUCTION}

Ce travail est une contribution à la démarche engagée par le Parc National de la Vanoise (PNV; Savoie) d'amélioration des connaissances liées au bâti traditionnel en cœur de Parc et de reconnaissance de sa valeur patrimoniale. Il s'inscrit dans le cadre de l'appel à propositions de partenariats scientifiques émis par le PNV en 2015. L'étude architecturale des bâtiments traditionnels situés en zone cœur du PNV (c.-à-d. la zone de haute protection de la nature qui s'étend sur $529 \mathrm{~km}^{2}$; figure $1 \mathrm{~b}$ ), menée par les architectes-collaborateurs P. Morard (en 2010) puis C. Iancovescu (entre 2011 et 2013), a fait état d'environ 400 bâtiments (Iancovescu, 2013; Bazan et Iancovescu, 2015). Cet inventaire a permis de mettre en avant un patrimoine - par ailleurs en voie de destruction par abandon ou parfois défiguré par des restaurations inadéquates (Brugière et al., 2000) - dans la perspective de sa valorisation et de sa protection en tant que témoin des sociétés qui ont vécu sur ces territoires au cours des siècles passés. Parallèlement, il a aussi posé la question de l'histoire, donc de la datation, de ce bâti remarquable.

L'interrogation sur les dates de construction est récurrente. Les dates disponibles reposent essentiellement sur les documents d'archives et sur les inscriptions datantes (chronogrammes) gravées sur les linteaux, les pierres d'angle et les poinçons des charpentes, en pignon aval des bâtiments. Ces informations donnent une certaine image de la dynamique démographique dans ces hautes vallées alpines. Mais les habitations sont très souvent construites en plusieurs étapes, modifiées ou réparées, avec un recours fréquent aux matériaux de remploi pour des raisons d'économie. Les dates recensées, bien qu'assez nombreuses, s'avèrent insuffisantes pour rendre compte à elles seules de l'histoire des bâtiments et de l'habitat sur la longue durée.

L'approche dendrochronologique permet de répondre avec une résolution temporelle fine (l'année) aux questions de l'ancienneté de l'habitat et de son évolution en termes de dynamique de construction, d'usage du bois et d'impact sur les milieux de montagne, la forêt en particulier. Dans les Alpes françaises, les recherches dendrochronologiques sur le bâti traditionnel ont surtout porté sur les Alpes du Sud (Édouard, 2010a; 2010b; Labbas, 2016; Shindo, 2016). Elles ont été réalisées sur la base des inventaires du bâti traditionnel du Service de l'Inventaire Général des Monuments et des Richesses Artistiques de la France, de documents cartographiques (cartes et cadastre anciens) et d'un échantillonnage pragmatique représentatif du bâti des hautes vallées (Briançonnais, Ecrins, Mercantour).

ArcheoSciences, revue d'archéométrie, 41(2), 2017, p. 7-22
Le Briançonnais étant une région des Alpes où le bois entre pour une grande part dans la construction traditionnelle des chalets et granges d'alpage (Blanchard, 1949-1956; Raulin, 1977a; Mallé, 1999; Pelletier, 2003), elle a été ciblée préférentiellement pour la mise en œuvre d'une telle approche. Ainsi, l'analyse de plus de 700 pièces de bois prélevées sur des constructions traditionnelles dans la vallée de la Clarée (commune de Névache, entre 1800 et 2000 m d'altitude) a apporté des données inédites sur l'histoire de l'habitat de montagne. Elle met en évidence une occupation humaine longue de plus de 800 ans, jalonnée d'épisodes de constructions identifiés dans la seconde moitié du XII ${ }^{e}$ siècle, à la fin du XIII ${ }^{e}$ siècle, dans la première moitié du XVI ${ }^{\mathrm{e}}$ siècle et au cours des XVIII ${ }^{\mathrm{e}}$ et XIX ${ }^{e}$ siècle (Édouard et Thomas, 2008; Édouard, 2010a; 2010b). Récemment, les recherches entreprises dans d'autres régions des Alpes du Sud, à savoir le massif du Mercantour (Labbas, 2016; dans le cadre du PIT Culture piloté par le Parc National du Mercantour) et la haute-Durance et les Préalpes de Digne (Shindo, 2016) étendent spatialement à l'ensemble des Alpes du Sud les résultats acquis dans la vallée de La Clarée. Elles contribuent à améliorer la connaissance de l'habitat montagnard et de l'usage du bois par les sociétés humaines depuis le Moyen Âge.

La présente étude vise à appliquer l'approche dendrochronologique à des chalets d'alpage situés en zone cœur du PNV (figure 1b), une région des Alpes du Nord qui n'a encore fait l'objet d'aucun travail de ce genre, même si des recherches sur l'habitat et l'architecture ont été réalisées par le passé (Onde, 1942; Blanchard, 1949-1956; Raulin, 1977b; Hermann, 1980; Dubois et al., 1994). Etant donné le nombre considérable de constructions identifiées, la stratégie de sélection des bâtiments étudiés s'est appuyée sur l'inventaire existant (Iancovescu, 2013) et a été menée en concertation avec le Parc et en lien avec les propriétaires.

\section{Sites D'ÉTUDE}

Dans le cadre de ce travail prospectif cinq bâtiments ont été sélectionnés pour représenter la diversité du bâti d'altitude présent dans le PNV (figure 1b):

- le secteur Plan du Lac-Entre-deux-Eaux (commune de Termignon) réunit un grand nombre de chalets d'alpage, l'ensemble le plus dense de la zone cœur, avec des bâtiments remarquables par leur architecture ou par leur regroupement en hameaux d'estive. Il a été proposé de travailler en priorité sur une ruine à la Civière (CIV), un chalet-exploitation d'alpage (parcelle n ${ }^{\circ}$ 146, 2092 m d'altitude);

- les bâtiments du vallon de Polset (POL; commune de Modane) sont considérés comme remarquables par le 
Figure 1 : (Voir planche couleur I) (a) Localisation du massif de la Vanoise et des chronologies de référence régionales utilisées dans le cadre de cette étude.

Toutes les chronologies de mélèze des Alpes françaises du Sud utilisées ne sont pas représentées (voir Tableau 1); (b) Localisation des bâtiments étudiés (triangle jaune) et des chronologies locales utilisées dans le massif de la Vanoise. Les surfaces glaciaires sont tirées de Gardent et al. (2014). Le fond topographique est un extrait de la BD ALTI de l'IGN (Projection Lambert 93).

Figure 1: (See colour plate I) (a) Location of the Vanoise massif in the Alps and of the regional tree-ring reference chronologies used in this study (coloured dots, by species).

(b) All larch chronologies from the southern French Alps used are not shown here (see Table 1); (b) Location of the studied buildings (yellow triangle) and of the local tree-ring chronologies used in the Vanoise massif (same key as in (a)). Red line marks the Vanoise National Park core area. Glacierized surfaces (light blue) are taken from Gardent et al. (2014). Background is hillshade-relief map based on the DEM BD ALTI from IGN (Lambert 93 Projection).

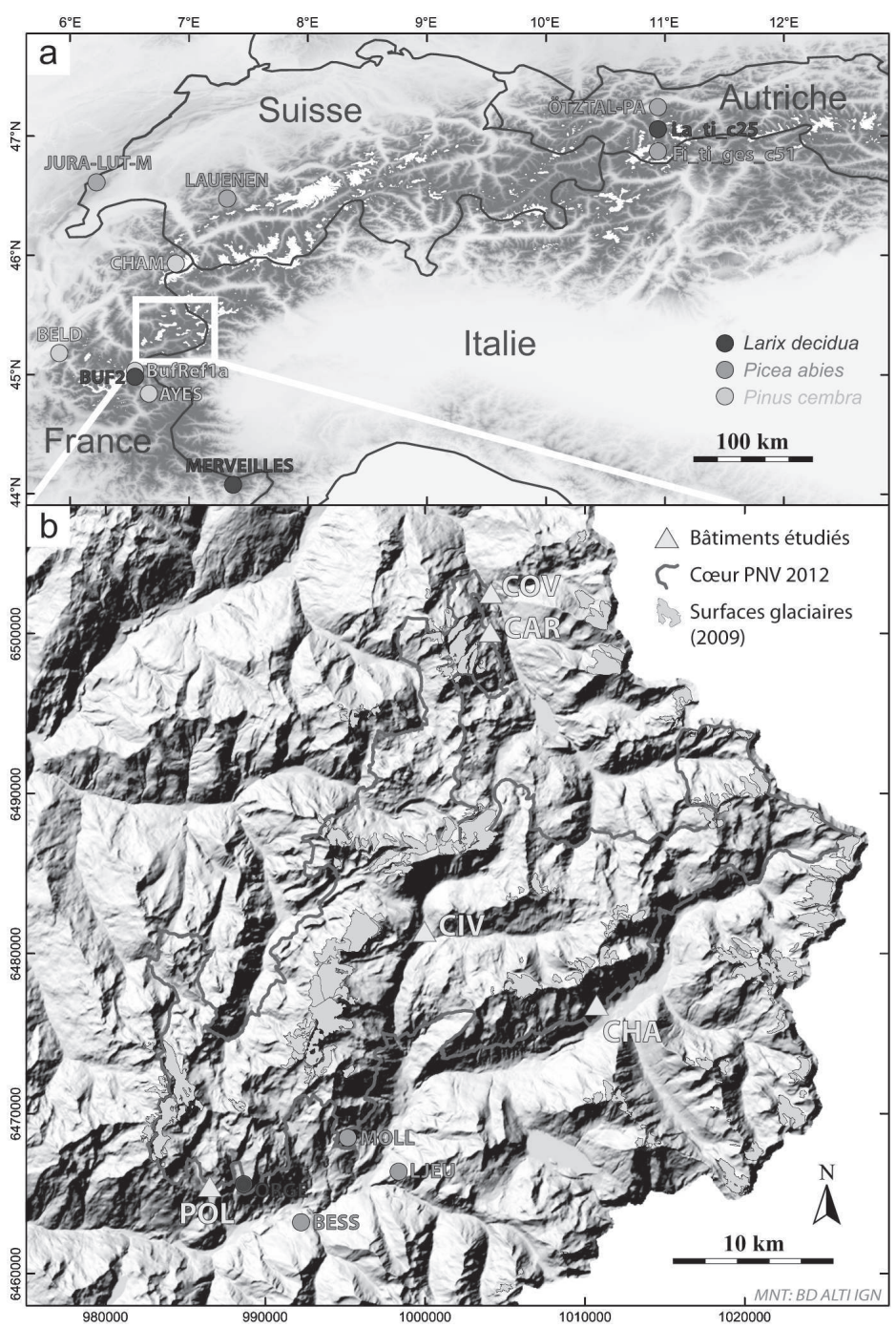

chaînage en bois des murs et par plusieurs chronogrammes (début et fin $\mathrm{XVIII}^{\mathrm{e}}$ siècle). Une grange a été étudiée sur ce site (parcelle $\mathrm{n}^{\circ}$ 192, $1860 \mathrm{~m}$ d'altitude);

- l'ensemble au Châtelard (CHA; commune de Lanslevillard) est unique à l'échelle du PNV en raison de ses dimensions et de son implantation en arc de cercle dans la pente. Les cinq bâtiments contigus du complexe ont été échantillonnés (parcelle 38, 2356 m d'altitude);

- la zone cœur située en Tarentaise offre moins de possibilités d'échantillonnage. Les chalets remarquables au Carroley (CAR; commune de Villaroger, parcelle 1718, $2122 \mathrm{~m}$ d'altitude) et au Covier (COV; commune de Sainte-FoyTarentaise, parcelle 1635-1636, $1300 \mathrm{~m}$ d'altitude) ont été retenus. Ce dernier est le seul à ne pas être un chalet d'alpage.

\section{Méthodologie}

\section{Échantillonnage}

Tous les bâtiments sélectionnés pour cette étude pilote étaient intentionnellement des chalets en ruine, principalement pour des raisons d'acceptation. L'échantillonnage a concerné toutes les pièces de bois qui possédaient une série de cernes suffisante ( $>60$ cernes environ) pour pouvoir envisager une datation dendrochronologique. Cela a représenté environ 20 à 30 pièces par bâtiment (à l'exception du Chalet au Covier). Deux modalités d'échantillonnage ont été mises en œuvre. Lorsque le contexte le permettait (bois déplacés, tombés, stockés), des sections entières ont été prélevées à la tronçonneuse. Dans le cas contraire, au moins deux carottes par pièce de bois ont été prélevées à l'aide d'une tarière de Pressler. 


\begin{tabular}{|c|c|c|c|}
\hline Nom & Période couverte & Localisation & Auteur/Ref. \\
\hline ORGE_Lade & $1353-1973$ & Forêt de l'Orgère, Modane, 73 & Tessier (1986) \\
\hline MERVEILLES_Lade & $933-1974$ & Vallée des Merveilles, Mercantour, 06 & Serre-Bachet (1978) \\
\hline BufRef1a_Lade & $1384-1894$ & Chalets de Buffère, Névache, 05 & J.-L. Édouard \\
\hline Dev2Dm1_Lade & $1394-1497$ & Chalets de Buffère, Névache, 05 & J.-L. Édouard \\
\hline Dev3Dm1_Lade & $1465-1860$ & Chalets de Buffère, Névache, 05 & J.-L. Édouard \\
\hline FOUI_Lade & $1669-1981$ & Fouillouse, Saint-Paul-les-Ubaye, 04 & F. Guibal \\
\hline CLAOUS_Lade & $1664-1990$ & Claous, Saint-Paul-les-Ubaye, 04 & F. Guibal \\
\hline TNX2_Lade & $1724-1990$ & Tournoux, Saint-Paul-les-Ubaye, 04 & F. Guibal \\
\hline LUD_Lade & $1577-1908$ & Grange de Bousieyas, St Dalmas-le-Selvage, 06 & J.-L. Édouard \\
\hline REFLade-JLE & $751-1995$ & $\begin{array}{l}\text { Moyenne de toutes les chronologies de mélèze } \\
\text { entre Névache et les Merveilles }\end{array}$ & $\begin{array}{l}\text { J.-L. Édouard; F. Guibal; Corona et al. } \\
\text { (2010) }\end{array}$ \\
\hline CHAM_Pice & $1262-2009$ & Vallée de Chamonix, 74 & Le Roy (2012) \\
\hline LJEU_Pice & $1744-1994$ & Bois du Jeu, Bramans, 73 & V. PetitColas \\
\hline BELD_Pice & $1172-1989$ & Lac Achard, Chamrousse, 38 & J.-L. Édouard \\
\hline AYES_Pice & $1475-1998$ & Bois des Ayes, Villard-St-Pancrace, 05 & J.-L. Édouard \\
\hline BUF2_Pice & $1594-2000$ & Chalets de Buffère, Névache, 05 & J.-L. Édouard \\
\hline LJEU_Pcab & $1546-1994$ & Bois du Jeu, Bramans, 73 & V. PetitColas \\
\hline MOLL_Pcab & $1729-1993$ & Forêt du Mollard Fleury, Aussois, 73 & V. PetitColas \\
\hline POUR_Pcab & $1807-1993$ & Lac des Pourettes, Chamrousse, 38 & V. PetitColas \\
\hline BESS_Pcab & $1720-1983$ & Forêt le Besseil, Avrieux, 73 & L. Tessier \\
\hline TERM_Abal & $1741-1983$ & Forêt de Termignon, Termignon, 73 & L. Tessier \\
\hline ÖTZTAL-PA & $1276-1974$ & Ref. régionale épicéa, Ötztal, Autriche & Siebenlist-Kerner (1984) \\
\hline LAUENEN & $1270-1949$ & Ref. régionale épicéa, Canton de Berne, Suisse & E. Schär \\
\hline JURA-LUT-M & $1299-2013$ & Réf. régionale épicéa Jura suisse & P. Gassmann \\
\hline La_ti_c25 & $741-2004$ & Ref. régionale Tyrol mélèze & K. Nicolussi \\
\hline Fi_ti_ges_c51 & $1006-2008$ & Ref. régionale Tyrol épicéa & K. Nicolussi \\
\hline
\end{tabular}

Tableau 1 : Chronologies de référence utilisées dans le cadre de cette étude.

L'espèce est indiquée dans le nom de la référence, Lade : Larix decidua Mill., mélèze; Pice : Pinus cembra L., pin cembro; Pcab : Picea abies (L.) Karst, épicéa; Abal : Abies alba Mill., sapin (Kaennel et Schweingruber, 1995).

Tableau 1: Tree-ring reference chronologies used in this study.

The species is indicated along with the name of the chronology, Lade: Larix decidua Mill., larch; Pice: Pinus cembra L., stone pine; Pcab: Picea abies (L.) Karst, spruce; Abal: Abies alba Mill., fir (Kaennel o Schweingruber, 1995).

\section{Mesure et interdatation}

Après leur préparation au laboratoire (ponçage ou surfaçage à la lame de rasoir afin d'obtenir une lisibilité parfaite) les échantillons ont été examinés sous loupe binoculaire et leur essence déterminée. La mesure de la largeur des cernes a ensuite été réalisée à l'aide d'une table de mesure LINTAB associée au logiciel TSAP-Win (Rinn, 2005), avec une précision de $0,01 \mathrm{~mm}$. Un minimum de deux rayons a été mesuré par pièce échantillonnée, mais souvent plus, notamment sur les sections entières. La moyenne des rayons mesurés sur une même pièce de bois donne une chronologie individuelle. Des synchronisations ont ensuite été recherchées entre les chronologies individuelles d'un même bâtiment, afin de constituer une chronologie moyenne de site. L'intercorrélation a été calculée comme indicateur de la force du signal commun entre les séries datées sur chaque site.

Dans un second temps, les séries individuelles et les chronologies de site ont été synchronisées avec des chronologies de référence - publiées ou non - disponibles pour les 
essences de résineux subalpines (figure 1a; tableau 1). Les tests de synchronisation ont été effectués avec le logiciel TSAP-Win (Rinn, 2005). Les indicateurs statistiques retenus sont le $t$ de Student modifié ( $t_{\mathrm{BP}}$; après standardisation des séries selon Baillie et Pilcher, 1973) et le pourcentage de coïncidence ou Gleichläufigkeit (Glk; Eckstein et Bauch, 1969). Le détail des résultats de l'interdatation est disponible par ailleurs (Le Roy et al., 2016).

Une date a été retenue lorsque les valeurs renvoyées sont supérieures aux seuils communément admis (e.g. $t_{\mathrm{BP}} \geq 3,5$ pour une série de 100 ans) et que l'examen visuel de l'ajustement sur la référence est bon. L'interdatation sur un réseau important de chronologies (figure 1a; Tableau 1) a permis d'évaluer la robustesse de certaines dates à l'échelle régionale, renforçant leur signification. L'hétéroconnexion a parfois été jugée pertinente, particulièrement s'agissant de chronologies locales.

\section{Lecture des résultats}

L'identification du dernier cerne formé (waldkante, WK, en allemand) est essentielle pour évaluer la qualité de la date obtenue par dendrochronologie. Celui-ci ne peut être identifié sans aucune ambigüité que si l'écorce est encore présente sur l'échantillon. Dans ce cas, il indique la date d'abattage à l'année près, voire à la saison près, si le cerne est parfaitement conservé (bois non altéré et non érodé). Dans le cas contraire, on détermine une date minimum pour l'abattage de l'arbre. Néanmoins dans certains cas, on peut, en l'absence d'écorce, raisonnablement estimer que le dernier cerne présent sur l'échantillon est le dernier cerne formé (par exemple si le dernier cerne de l'échantillon est traçable sur une grande partie de la circonférence d'une section). Cela est plus délicat à déterminer lorsque seules des carottes sont disponibles pour analyse.

La nomenclature utilisée pour la présentation des résultats est la suivante :

- WKL : le dernier cerne présent est le dernier cerne formé (se termine par du bois final, complet ou non). La présence de l'écorce est indiquée par un 'B';

- WKE : le dernier cerne présent est le dernier cerne formé (se termine par du bois initial);

- (WK) : le dernier cerne présent est proche du dernier cerne formé. Il peut aussi être le dernier cerne formé, mais une incertitude demeure;

- 1837+ : date minimum pour l'abattage de l'arbre. Le nombre de cernes manquants jusqu'au dernier cerne formé est inconnu (ce nombre peut aussi être nul). Lorsque l'aubier n'est pas présent (ou non décelable) on parle d'un terminus post quem pour la mort de l'arbre, c.-à-d. la date à partir de laquelle l'arbre a pu être abattu. Dans ce cas, pour le mélèze, un nombre de cernes minimal d'aubier (14) a été ajouté au dernier cerne de duramen détecté pour proposer un terminus post quem plus proche de la date d'abattage réel. C'est cette date qui est utilisée dans la suite de l'article. À l'inverse, lorsque l'aubier est identifiable et manifestement incomplet ( $<15$ cernes), une estimation du nombre maximal de cernes d'aubier a été effectuée pour obtenir un intervalle temporel susceptible d'inclure la date d'abattage. Cette estimation se base sur des comptages du nombre de cernes d'aubier chez le mélèze effectués par Shindo (2016).

L'estimation du nombre de cernes manquants jusqu'à la moelle (pith offset) a également été réalisée sur les carottes lorsque celle-ci était absente. Ceci afin d'avoir une appréciation plus juste de l'âge des arbres abattus. Cette estimation repose sur des courbes de croissance établies pour le mélèze et le pin cembro dans les Alpes du Sud (J.-L. Édouard et F. Guibal, données non publiées).

Lidentification des pièces de bois qui appartenaient à un même arbre d'origine a été tentée. Les résultats sont donnés à titre indicatif, la difficulté résidant dans le choix des seuils à retenir pour identifier ces échantillons (des mesures effectuées sur des secteurs éloignés d'une même tige peuvent renvoyer des valeurs semblables aux corrélations calculées entre deux arbres différents). Les seuils néanmoins retenus correspondent approximativement à des valeurs de $18>t_{\mathrm{BP}}$ $>14$ pour le mélèze, et de $14>t_{\mathrm{BP}}>10$ pour l'épicéa.

\section{Résultats}

\section{Ruine à La Civière (CIV)}

Ce bâtiment présente le chronogramme le plus ancien recensé dans la zone cœur du PNV (Iancovescu, 2013) : le linteau en bois de la porte du pignon sud-est est gravé ' 1471' (figure 2c). Cette date inscrite en chiffres arabes s'avère exceptionnelle à l'échelle régionale. En effet, en proche vallée d'Aoste, les plus anciennes dates gravées remontent au XVI ${ }^{\mathrm{e}}$ s, à une exception près (C. Remacle, comm. pers., 2016). Sur ce bâtiment, d'autres dates gravées existent sur des pierres retournées (c.-à-d. réutilisées) : ' 1546 ' sur une pierre du chaînage d'angle nord-est (figure 2d), et '1666' sur une pierre du même mur à l'intérieur du bâtiment. Cette dernière se situe à $1,5 \mathrm{~m}$ du mur nord et $1,5 \mathrm{~m}$ du sol (figure 2e).

L'échantillonnage a concerné vingt-huit pièces de bois. Il a porté en premier lieu sur les rares pièces encore en place, qui ont été sondées à la tarière. Il s'agit des linteaux de la porte du pignon sud-est (trois pièces, CIV01 à 03 ; le 01 

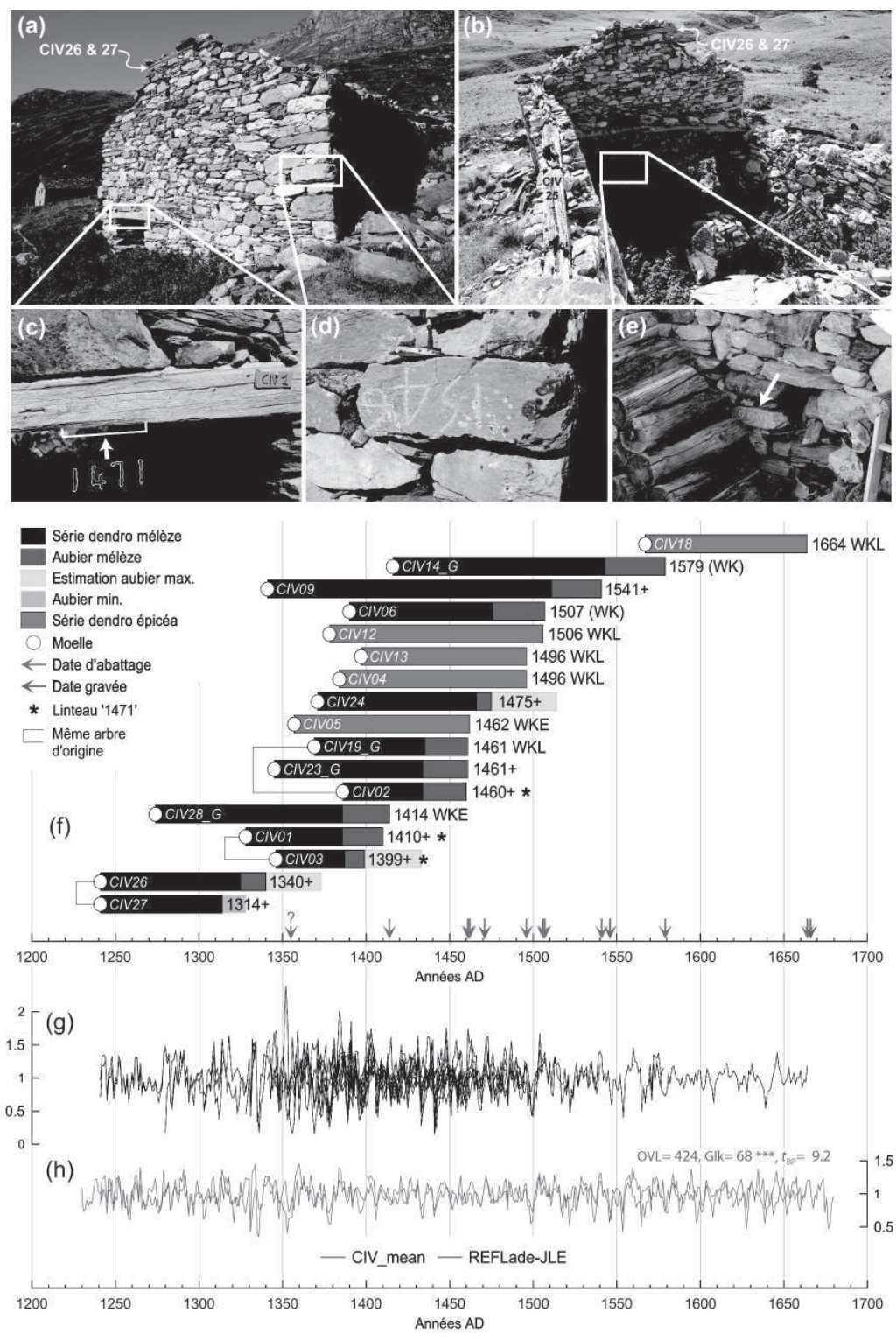

Figure 2 : (Voir planche couleur II) Ruine à La Civière (2092 m), parcelle 146 .

(a) Vue d'ensemble du pignon sud-est (cliché: 07.09.15); (b) Vue vers le sud-est depuis le mur nord. Le tas de bois échantillonné est au dos du pignon; Chronogrammes : (c) '1471'; (d) '1546' retourné; (e) '1666' retourné; (f) Résultats des datations dendrochronologiques effectuées sur la ruine à la Civière. La nomenclature utilisée pour la présentation des dates d'abattage est détaillée dans le texte (Section 3. Lecture des résultats); (g) Séries individuelles indicées à l'aide d'une moyenne mobile sur 11 ans; (h) Moyenne de site CIV (mélèze et épicéa) en position de synchronisation sur la moyenne des références de mélèze des Alpes du Sud et résultats des tests statistiques.

Figure 2: (See colour plate II) Ruin at La Civière (2092 $m$ a.s.l.), parcel 146.

(a) Overview of the southeastern gable wall (photograph: 07.09.15); (b) View towards southeast from the northern wall. Sampled wood pile is on the back of the southeastern gable wall; Building date inscriptions: (c) '1471'; (d) '1546' overturned; (e) '1666' overturned; (f) Results of tree-ring dating performed on the ruin at La Civiere. Nomenclature used for the presentation of felling dates is detailed in main text (Section 3. Lecture des résultats); (g) Individual series indexed using a 11-year moving average; (h) CIV site mean series (larch and spruce) in crossdating position on a reference that consists of several southern French Alps larch chronologies. Crossdating results are also shown. étant la pièce gravée ' 1471 '), des deux pièces de chaînage de ce même pignon (CIV26 et 27 ; figure $2 \mathrm{a} / \mathrm{b}$ ) et de la panne sablière coiffant le mur nord (CIV25; figure 2b). Toutes les autres pièces avaient été déplacées, et ont donc été prélevées à la tronçonneuse. Dans la partie nord-ouest du bâtiment, les poutres reposant sur le sol ont toutes été échantillonnées (CIV20 à 24). Le reste des échantillons a été prélevé dans le tas de bois établi par le propriétaire derrière le pignon sudest (CIV04 à 19 et CIV28; figure 2b). Certains peuvent être identifiés comme des linteaux de porte (e.g. CIV06), mais la majorité sont des tronçons de pannes. L'échantillonnage des rondins sciés ayant été quasi exhaustif il s'est avéré que de nombreux échantillons appartenaient aux mêmes arbres d'origine. Des regroupements par arbre ont donc été faits pour les pièces qui n'étaient plus en place. Ces groupements sont signalés par le suffixe "_G" dans les résultats (figure $2 \mathrm{f}$ ).

Deux essences ont été utilisées sur le site de La Civière : le mélèze (69\% des arbres échantillonnés) et l'épicéa (31\%). Le résultat des datations est présenté sur la figure $2 \mathrm{f}-\mathrm{h}$. Nous n'avons pas obtenu de résultats pour trois échantillons (représentant deux arbres). L'intercorrélation des séries datées est de 0,58 et 0,39 pour les deux espèces présentes, respectivement. L'âge moyen des arbres lors de leur abattage était de $119 \pm 31$ ans $(\mathrm{n}=14)$. Les bois d'épicéa ont été les plus difficiles à dater du fait de l'absence de références longues dans le massif. Ils ont cependant été synchronisés sur des références régionales du Jura, de Suisse centrale et d'Autriche avec des résultats très fiables (Le Roy et al., 2016). 
La première période d'abattage n'est pas datée précisément. Il s'agit des bois du chaînage du mur est (figure $2 \mathrm{a} / \mathrm{b}$ ). Ces deux pièces (CIV26 et 27) appartiennent au même arbre d'origine. Le début de l'aubier n'est présent que sur CIV26 (premier cerne : 1325) dont la date d'abattage est fixée à $1340+$. Selon les estimations, la date d'abattage réelle de cet arbre est antérieure à 1373 et pourrait se situer aux alentours de 1355 (figure 2f). En effet, le comptage des cernes d'aubier effectué sur les mélèzes de ce bâtiment dont le dernier cerne est présent donne $31 \pm 4$ cernes $(n=4)$.

La seconde période d'abattage est datée d'après le bois CIV28_G dont le dernier cerne est présent mais incomplet (absence de bois final), indiquant un abattage au printemps/ début d'été 1414. Les deux pièces du linteau CIV01 et 03 (provenant du même arbre) ne portent pas le dernier cerne et la date minimum de $1410+$ pour la mort de cet arbre est cohérente avec un abattage contemporain de celui du CIV28_G.

La troisième période d'abattage est contrainte par trois arbres différents. Le dernier cerne formé n'a pu être identifié sans ambigüité que sur un seul des échantillons de ce groupe, CIV05, dont l'abattage date du printemps/début été 1462 (absence de bois final). Néanmoins, le dernier cerne formé paraît également être présent sur CIV19_G. Les comptages faits sur de nombreux rayons et sur deux sections différentes (CIV19 et 20) coïncident. Il est donc peu probable qu'un cerne externe manque de façon uniforme sur cet échantillon, dont la mort daterait de ce fait de 1461 (le bois final est quasi complet à complet, donc un abattage intervenant tard dans la saison de croissance ou l'hiver suivant). Le dernier cerne conservé sur le troisième arbre, CIV23_G, est celui de 1461 ; malheureusement il est très érodé, ce qui ne permet pas d'affirmer qu'il s'agit du dernier cerne formé, même si cela est possible. En conclusion, il semble qu'il y ait ici deux dates d'abattage distinctes mais rapprochées : fin de l'année 1461 pour les mélèzes et printemps/début d'été 1462 pour l'épicéa (figure 2f).

La quatrième période d'abattage est représentée par deux (ou trois) arbres. Les deux épicéas CIV04 et CIV13 présentent tous les deux le dernier cerne formé et indiquent un abattage en 1496 (le bois final est quasi complet à complet, donc un abattage intervenant entre la fin d'été et la fin de l'hiver suivant). Le mélèze CIV24 présente seulement les dix premiers cernes d'aubier (premier cerne d'aubier : 1466). Il est donc possible qu'il ait fait partie de la même phase d'abattage que les deux épicéas en 1496 (figure 2f).

Une cinquième phase peut être identifiée d'après deux bois. L'épicéa CIV12 a été abattu en 1506 (le dernier cerne formé est présent sans ambigüité, il est quasi complet à complet, donc un abattage intervenant entre la fin de l'été 1506 et la fin de l'hiver suivant). Le mélèze CIV06 a vraisemblablement été abattu en 1507 (une incertitude demeure sur le fait que le dernier cerne présent est le dernier cerne formé. De plus, un peu de bois final est présent mais il n'est pas possible de dire s'il est complet ou non). On est à nouveau ici en présence de deux dates d'abattage proches, mais distinctes (figure 2f).

Les dates de mort sont ensuite isolées et il n'est pas possible de déterminer des phases ayant impliqué plusieurs arbres (figure 2f). Elles sont intervenues en 1541+ (le dernier cerne présent est très érodé), en été 1579 (le dernier cerne présent est très érodé, incertitude sur le fait qu'il s'agit bien du dernier cerne formé, quelques cellules de bois final sont visibles) et en 1664 (le dernier cerne formé est présent sans ambigüité, et complet, donc abattage intervenant entre la fin de l'été et la fin de l'hiver suivant).

La confrontation des données dendrochronologiques et des trois chronogrammes identifiés sur le bâtiment montre qu'au moins une date d'abattage a été identifiée à chaque fois dans la décennie précédant la date gravée (figure 2f) : les dates 1461/62 peuvent ainsi être mises en relation avec l'inscription '1471', la date $1541+$ avec l'inscription '1546' et la date 1664 avec l'inscription '1666'.

\section{Grange au Polset (POL)}

Un des aspects notables de ce bâtiment est le chainnage exceptionnel sur trois niveaux dans le pignon ouest. Le niveau inférieur se prolonge sur trois façades différentes (figure 3a). L'arrondi du pignon est (amont) (figure 3e), enterré, est également remarquable. Deux chronogrammes existent sur la grange : ' $17 \dagger 24$ ' gravé en pignon ouest sur une pierre de chaînage d'angle (figure 3c), et une date ' $17 \nmid 24$ ' moins lisible, gravée sur le chaînage inférieur, au-dessus de la porte d'accès à la cave en façade sud (figure $3 \mathrm{~d}$ ).

Dix-sept pièces de bois ont été échantillonnées à la tarière. Toutes les pièces principales ont été sondées.

L'essence utilisée pour ce bâtiment est très majoritairement le mélèze, qui représente tous les échantillons prélevés, à l'exception d'un chevron (épicéa). Le résultat des datations est présenté sur la figure $3 \mathrm{f}-\mathrm{h}$. Tous les échantillons sauf la panne sablière nord (POL06) ont pu être datés. L'intercorrélation des séries de mélèze datées est de 0,74 , une valeur particulièrement élevée. L'âge moyen des arbres de la charpente lors de leur abattage était de $138 \pm 26$ ans $(\mathrm{n}=11)$. Le chevron et la cale échantillonnés n'ont pas été intégrés à cette moyenne. Les datations de ces deux pièces ne figurent pas sur la figure $3 \mathrm{f}$ car elles restent à confirmer en raison de séries courtes. Néanmoins, le chevron (POL07, 52 cernes) pourrait avoir été abattu en 1723 (le dernier cerne formé est présent). Le 

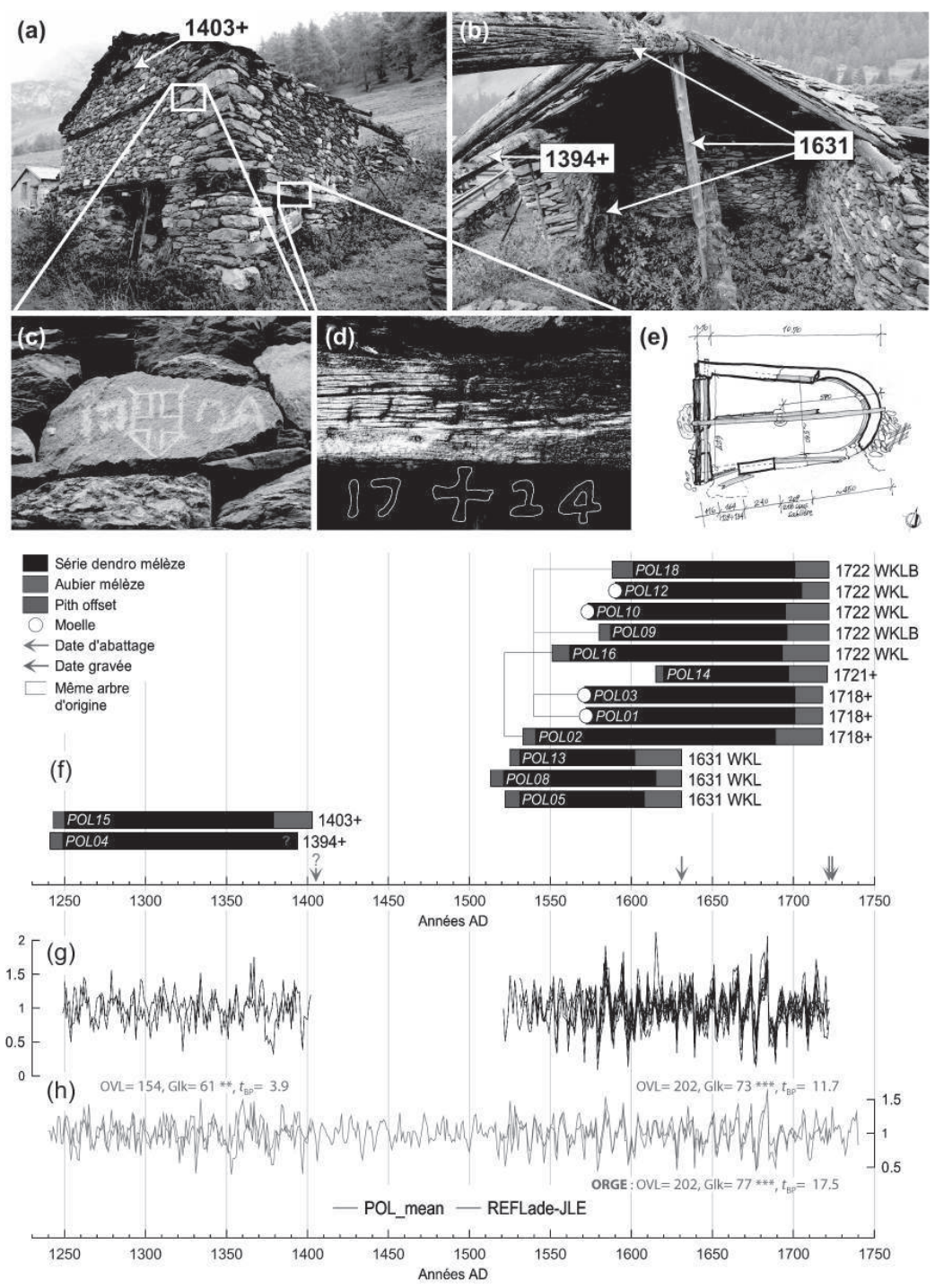

Figure 3 : (Voir planche couleur III) Grange au Polset (1860 m), parcelle 192 .

(a) Vue d'ensemble du pignon ouest et du mur sud avec la localisation des chronogrammes (cliché: 23.09.15). Le résultat de la datation de la pièce du chaînage supérieur (en remploi) est indiqué; (b) Vue du bâtiment depuis l'amont, à l'extrémité est de la panne faîtière. Les datations des pièces de remploi sont indiquées (panne sablière, poteau central, panne faîtière et panne supportant le plancher); (c) Inscription '1724' avec le blason de la Savoie; (d) Inscription '1724' gravée sur le linteau où a été prélevé l'échantillon POL03; (e) Plan de la grange (levés et dessin: C. Iancovescu) ; (f) Résultats des datations dendrochronologiques effectuées sur la grange au Polset. La nomenclature utilisée pour la présentation des dates d'abattage est détaillée dans le texte (Section 3.3); (g) Séries individuelles indicées à l'aide d'une moyenne mobile sur 11 ans; (h) Moyenne de site POL en position de synchronisation sur la moyenne des références de mélèze des Alpes du Sud et résultats des tests statistiques. Les résultats sur la chronologie locale de mélèze de l'Orgère (Modane, Maurienne) sont également indiqués (bas).

Figure 3: (See colour plate III) Barn at Polset $(1860 \mathrm{~m}$ a.s.l.), parcel 192.

(a) Overview of the western gable wall and of the southern wall with the location of the building date inscriptions (photograph: 23.09.15). Tree-ring date of one of reuse wood pieces is shown; (b) View of the building taken from upstream, at the eastern end of the ridge beam. Tree-ring dates of reuse pieces are indicated (lower purlin, central post, ridge beam and the beam supporting the floor); (c) Inscription '1724' with the Arms of the House of Savoy; (d) Inscription '1724' carved on the lintel where sample POLO3 was taken; (e) Floor plan of the barn (survey and sketch: C. Iancovescu); (f) Results of tree-ring dating performed on the barn at Polset. Nomenclature used for the presentation of felling dates is detailed in main text (Section 3.3); (g) Individual series indexed using a 11-year moving average; (h) POL site mean series in crossdating position on a reference that consists of several southern French Alps larch chronologies. Crossdating results are shown both on this regional reference and on the Orgère local larch reference (Modane, Maurienne).

prélèvement d'autres chevrons permettrait de déterminer si cette date est correcte. La seconde date ne marque pas à proprement parler une phase de construction. Il s'agit d'une cale (POL17, 62 cernes) située entre l'étai et la panne POL13 et dont la date d'abattage a été fixée à 1818 (le dernier cerne formé est présent).

En ce qui concerne les bois de charpente datés avec sécurité, trois périodes d'abattage distinctes ont été identifiées (figure 3f). La première phase n'est pas datée précisément mais est intervenue au tout début du Xve siècle d'après les échantillons POL04 et POL15. En raison de l'altération du bois, l'aubier n'a pu être identifié sur POL04, alors que 25 cernes ont été comptés sur POL15 (premier cerne d'au- bier en 1379). La moyenne des cernes d'aubier comptés sur les échantillons de la grange étant relativement faible $(25 \pm$ 5 cernes; $\mathrm{n}=8$ ), on peut estimer que la date d'abattage réelle de POL15 n'est pas très éloignée de la date minimum déterminée à $1403+$ (figure 3f).

La deuxième phase est renseignée par trois échantillons provenant de trois arbres différents (POL05, POL08, POL13). Leur analyse indique un abattage entre la fin de l'été 1631 et la fin de l'hiver suivant, le bois final du dernier cerne étant quasi complet à complet (figure 3f).

La dernière phase d'abattage est la mieux représentée. Les échantillons qui portent le dernier cerne de manière indiscutable indiquent un abattage entre la fin de l'été 1722 et la fin 
de l'hiver suivant. La lisibilité n'est pas suffisante sur la majorité des échantillons pour déterminer si le cerne 1722 est achevé, mais le bois final est présent à chaque fois. Parmi les échantillons datés, deux portaient encore l'écorce (POL09 et POL18). La confrontation avec les dates gravées indique que les bois abattus ont été stockés deux ans avant leur mise en ouvre dans la construction de la grange (figure $3 \mathrm{f}$ ).

Pour trois échantillons, la date d'abattage a été fixée à $1718+$ sans qu'il soit possible de déterminer avec certitude s'il s'agit du dernier cerne ou non (figure 3f). L'un de ces échantillons, POL02, semble appartenir au même arbre que POL16 $\left(t_{\mathrm{BP}}=17,5\right)$, ce qui pourrait indiquer une date d'abattage commune en 1722. En revanche, le dernier cerne identifié sur tous les rayons mesurés sur les deux échantillons POL01 et POL03 - qui semblent appartenir au même arbre $\left(t_{\mathrm{BP}}=17,3\right)$ - est le même, celui de 1718 . Or, la série moyenne de ces deux échantillons corrèle très fortement avec d'autres échantillons dont l'abattage est daté de 1722 avec certitude, comme POL09 $\left(t_{\mathrm{BP}}=14,8\right)$ ou POL18 $\left(t_{\mathrm{BP}}=13,8\right)$. Sur ce site, la très grande homogénéité des séries dendrochronologiques issues des arbres abattus au XVIII ${ }^{\mathrm{e}}$ siècle (figure $3 \mathrm{~g}$ ) - approchant des valeurs voisines de celles d'échantillons provenant d'un même arbre - indique vraisemblablement des arbres ayant poussé dans des conditions stationnelles identiques. Il est donc possible qu'un arbre ait été abattu en 1718 (POL01/03). Comme attendu, les résultats de l'interdatation de ces arbres sur la chronologie de référence de l'Orgère, distante de $2 \mathrm{~km}$ seulement (figure $1 b$ ), sont extrêmement hauts (figure $3 h$ ).

En conclusion, le remploi d'au moins deux générations de bois est manifeste sur ce bâtiment reconstruit pour la dernière fois au XVIII ${ }^{\mathrm{e}}$ siècle. En effet, trois pièces majeures datent du XVII ${ }^{\mathrm{e}}$ siècle et deux autres pièces datent du début $\mathrm{du} \mathrm{Xv}^{\mathrm{e}}$ s. L'hypothèse de bois anciens ramenés du village pour remploi à l'alpage est faible compte tenu de la présence sur ce site d'une forêt de mélèze pouvant être utilisée comme bois d'œuvre.

\section{Ensemble au Châtelard (CHA)}

L'ensemble se présente comme la juxtaposition de 5 bâtiments (numérotés I à V, de l'aval à l'amont; figure 4a). La grange située en contrebas de cet ensemble n'a pas fait l'objet de prélèvements. D'après l'analyse architecturale, le bâtiment $V$, très dégradé, semble être le plus ancien. Ensuite, le bâtiment I aurait été construit contre le bâtiment II (fenêtre occultée), lui-même construit contre le bâtiment III (faîtière du II sur corbeau du pignon du III). Ce dernier serait donc le premier construit de l'alignement. Le bâtiment IV aurait été rajouté postérieurement pour combler l'espace vide entre l'alignement et le bâtiment V. En effet, la ligne de jonction entre le III et le IV, en façade, suggère un démantèlement partiel du III pour refaire le chaînage d'angle lié au IV. De plus, le bâtiment IV possède une maçonnerie très soigneusement exécutée (niches, hotte de cheminée), signe d'une amélioration des techniques. Enfin, il est possible que toutes ces dates de construction soient relativement proches dans le temps.

Plusieurs chronogrammes existent sur des pierres retournées : ' 1623 ' sous la porte-fenêtre percée dans le pignon sud du bâtiment II (figure 4c), '1672' en façade sud-est du bâtiment III (figure 4a), ainsi que sur des pierres à l'endroit : '1787' (alternatives : 1782 ou 1797?) sur le chaînage d'angle ouest du bâtiment I (figure 4b), et '1801' sur le chaînage d'angle sud-est du bâtiment V. Des pièces de bois gravées sont également présentes : ' $16 \nmid 70$ ' sur un linteau intérieur dans le mur de refend de la grange inférieure non échantillonnée, et ' $17 \nmid 6$ ?' ou ' $17 \nmid 76$ ' sur le linteau de la porte située en façade sud-est du bâtiment IV (figure 4d).

Vingt-trois pièces de bois ont été échantillonnées à la tarière et à la tronçonneuse. Toutes les pièces principales ont été sondées.

Deux essences ont été employées sur ce site : le mélèze (64\% des échantillons) et l'épicéa (36\%). Le résultat des datations est présenté sur la figure 4e-h. Nous n'avons pas obtenu de résultat pour 5 pièces sur les 23 pièces échantillonnées. Il s'agit de la faittière du bâtiment I et de tous les échantillons du bâtiment III (hormis la cave). L'intercorrélation des séries datées est de 0,55 et 0,54 pour les deux espèces présentes, respectivement. L'âge moyen des arbres lors de leur abattage était de $108 \pm 29$ ans $(\mathrm{n}=21)$.

Les dates les plus anciennes concernent le bâtiment $\mathrm{V}$, le plus dégradé. Deux pannes écroulées ont été échantillonnées. Les dates obtenues sont assez éloignées - début $\mathrm{Xv}^{\mathrm{e}}$ et fin $\mathrm{XVI}^{\mathrm{e}}$ siècle - rendant difficile une interprétation (figure 4e). Tout au plus peut-on émettre l'hypothèse que cette partie en ruine n'a pas connu de réfection par la suite et que la pièce la plus ancienne est d'origine.

Une deuxième période d'abattage peut être identifiée au début du XVII ${ }^{\mathrm{e}}$ siècle avec deux pièces du bâtiment II appartenant au même arbre d'origine. Il s'agit de la panne faîtière et du linteau intérieur de la porte-fenêtre qui donne sur le bâtiment I (figure 4c). On peut éventuellement rattacher à cette période d'abattage du début du XVII ${ }^{e}$ siècle la panne CHAV.1 du bâtiment $\mathrm{V}$.

Ensuite, le bâtiment IV est bien contraint par trois dates concordantes au milieu du XVIII ${ }^{\mathrm{e}}$ siècle. La date d'abattage de la panne faîtière (CHAIV.2) est fixée à $1757+$ et celle du linteau de la porte en façade est à 1756+ (figure 4e). Le dernier cerne formé n'a pas été identifié sur ces pièces mais 

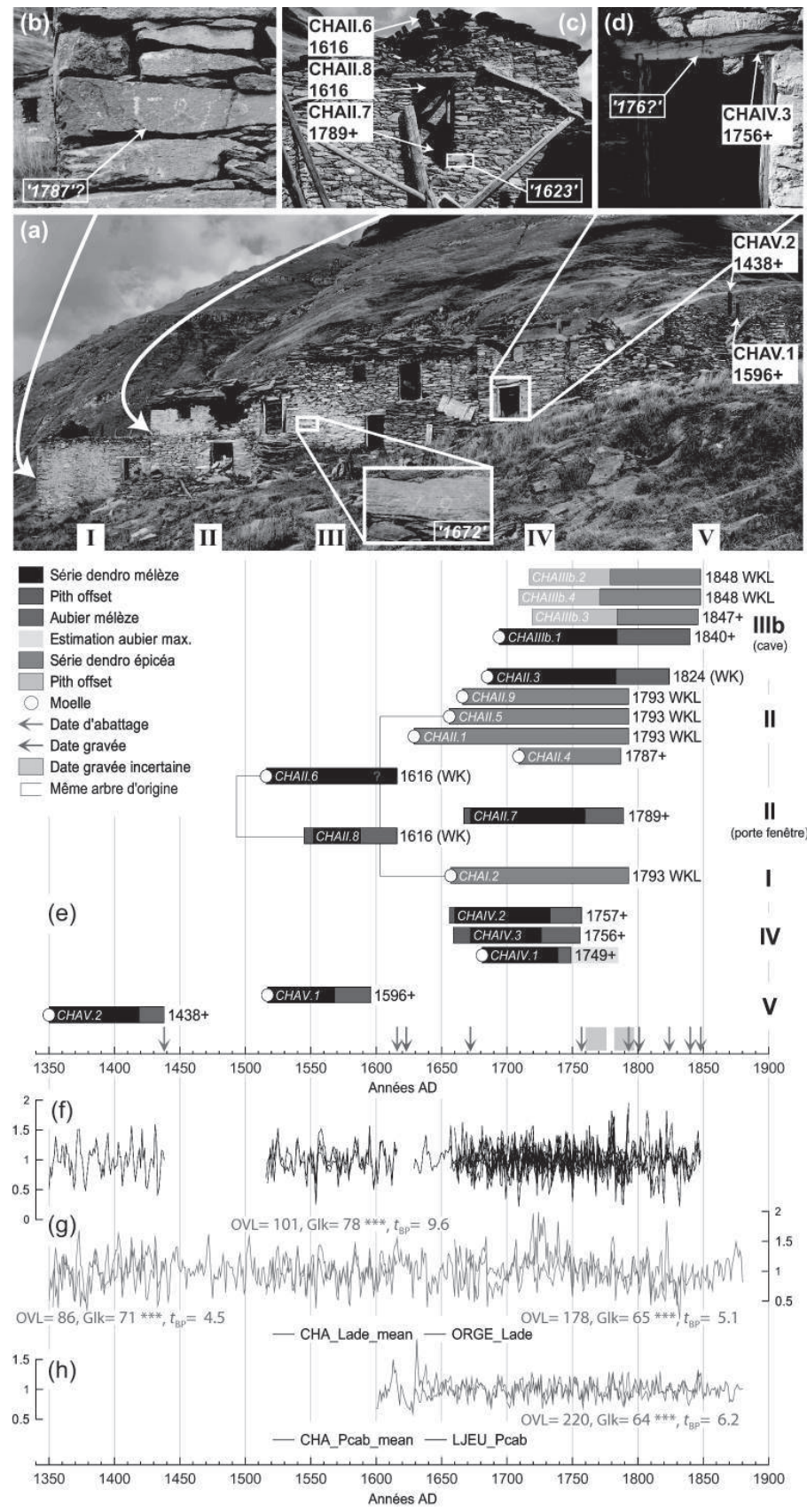

l'aubier comptait 24 et 30 cernes, respectivement. Ces dates sont cohérentes avec l'inscription présente sur le linteau de la porte en façade est qui n'a pu être déchiffrée avec certitude : "176?" ou éventuellement "1776" (figure 4d).

La période d'abattage majeure identifiée sur l'ensemble au Châtelard intervient à la fin du XviII ${ }^{e}$ siècle (figure 4e). Elle concerne des pièces des bâtiments I et II. Les échantillons qui conservent le dernier cerne formé indiquent un abattage en 1793 (bois final présent et quasi complet à complet). Ce sont principalement les poutres supportant le
Figure 4 : (Voir planche couleur IV) Ensemble au Châtelard (2356 m), parcelle 38 .

(a) Vue générale en direction de l'ouest avec l'emplacement des différents chronogrammes (cliché: 10.09.15). Le résultat des datations réalisées sur le bâtiment $V$ est indiqué; (b) Inscription du pignon sud du bâtiment I, '1787' (alternatives : '1792', '1797'); (c) Pignon sud du bâtiment II avec l'emplacement du chronogramme retourné et le résultat des datations effectuées sur la panne faîtière et sur la porte-fenêtre donnant sur le bâtiment I; (d) Linteau gravé de la porte sud-est du bâtiment IV, l'inscription est '176?' (alternative : '1776'). La datation dendrochronologique de cette pièce est 1756+; (e) Résultats des datations dendrochronologiques effectuées sur l'ensemble au Châtelard. Les séries datées sont regroupées par bâtiments (chiffres romains à droite). La nomenclature utilisée pour la présentation des dates d'abattage est détaillée dans le texte (Section 3.3); (f) Séries individuelles indicées à l'aide d'une moyenne mobile sur 11 ans; Moyennes de site CHA, par essences, en position de synchronisation sur les chronologies de référence locales : Orgère, mélèze (g) et LJEU, épicéa (h) et résultats des tests statistiques.

Figure 4: (See colour plate IV) Building complex at Châtelard (2356 m a.s.l.), parcel 38 .

(a) Overview westward with the location of various building date inscriptions (photograph: 10.09.15). Tree-ring dates from building $V$ are also shown; (b) Inscription located on the building I southern gable wall, '1787' (alternatives: '1792', '1797'); (c) Building II southern gable wall with the location of the overturned building date inscription. Tree-ring dates from the ridge beam and from the door opening on building I are also indicated; (d) Carved lintel of the southeastern door of building IV, inscription is '176?' (alternative: '1776'). Tree-ring date of this piece is 1756+; (e) Results of tree-ring dating performed on the building complex at Chatelard. Dated tree-ring series are grouped by building (roman numerals on the right). Nomenclature used for the presentation of felling dates is detailed in main text (Section 3.3); (f) Individual series indexed using a 11-year moving average; CHA site mean series for each species in crossdating position on local reference chronologies: Orgère, larch (g) and LJEU, spruce (h). Crossdating results are also shown. plancher du bâtiment II (épicéa), qui ne sont plus en place (à l'exception de CHAII.9). D'autres pièces pour lesquelles un terminus post quem a été déterminé peuvent se raccorder à cette date : le seuil extérieur de la porte-fenêtre (1789+) et le linteau intérieur de la fenêtre en façade est (1787+). La panne CHAII.3 a une date d'abattage bien distincte et postérieure (1824) alors qu'elle a été échantillonnée parmi les autres pannes du bâtiment II. Sa position initiale n'est pas connue, mais elle avait une orientation longitudinale au sol, et non transversale comme les autres. 
Enfin, les bois les plus récents sont ceux de la cave du bâtiment III. La panne du toit est datée de 1840+ (le dernier cerne formé paraît être présent, mais les cernes externes sont très fins, peu lisibles et comportent des cernes absents). Les planches de volige proviennent d'arbres abattus en 1848 (épicéa; écorce présente). Il s'agit donc, au milieu du XIX $x^{e}$ siècle, de la dernière phase de construction/réparation sur le site du Châtelard (figure 4e). Le bâtiment III est le mieux préservé de l'ensemble, néanmoins les pièces maitresses de ce bâtiment n'ont pas pu être datées. Bien que l'essence utilisée ici soit le mélèze et malgré le fait que les séries soient synchrones (date d'abattage identique pour la faîtière, le poteau central et deux des solives; dernier cerne formé présent sur toutes les pièces), aucune date fiable n’a encore été déterminée pour ces échantillons.

L'ensemble au Châtelard est extrêmement composite et il n'a pas été possible de relier des dates d'abattage avec des chronogrammes de façon claire (figure 4e). Malgré tout, les deux pièces du bâtiment II datées 1616 semblent pouvoir être rapprochées de l'inscription retournée '1623' identifiée à proximité (figure 4c). Ces trois éléments ont ensuite été repris dans la réfection du bâtiment à la fin du XviII siècle (figure 4e). Sur le bâtiment IV, la date de 1756+ déterminée pour le linteau de la porte semble également concorder avec l'inscription gravée qui y figure, '176?' (ou éventuellement '1776') (figure 4d/e).

Les résultats de l'analyse dendrochronologique confirment en partie les hypothèses émises quant aux étapes successives de réparation-extension - visibles par les techniques et les mises en œuvre (le bâtiment IV semblait être le plus récent, ou le plus soigné) - dont la dernière phase majeure date de la fin du XVIII ${ }^{e}$ siècle. Cependant, selon la configuration de l'ensemble, une construction initiale simultanée des bâtiments est à retenir comme probable (au XV siècle? XVII ${ }^{e}$ siècle?). Nos données confirment également l'abandon précoce du bâtiment $\mathrm{V}$, ce qui permet d'appréhender le niveau de dégradation d'une ruine par rapport à l'ancienneté de l'abandon.

\section{Chalet au Carroley (CAR)}

L'intervention sur ce bâtiment a été réalisée avant la phase de travaux prévue pour l'été 2016, qui a impliqué la dépose de la charpente actuelle (figure 5a/d). Aucun chronogramme n'a été identifié sur ce bâtiment.

Vingt-et-une pièces de bois ont été échantillonnées à la tarière. Toutes les pièces principales ont été sondées.

L'essence utilisée sur ce site est exclusivement l'épicéa. Le résultat des datations est présenté sur la figure $5 \mathrm{e}-\mathrm{g}$. Les séries contemporaines corrèlent entre elles de façon significative (intercorrélation : 0,58). L'âge moyen des arbres lors de leur abattage était de $106 \pm 29$ ans $(n=17)$.
Deux échantillons sont plus vieux que la majorité, avec des dates d'abattage dans la première moitié du XviI ${ }^{\mathrm{e}}$ siècle (1632+; CAR18) et dans la première moitié du XviII ${ }^{\mathrm{e}}$ siècle (1721+; CAR16). Il s'agit respectivement de la pièce maçonnée dans le pignon ouest (figure 5c), dont la fonction n'est pas connue (support de cloison ou de meuble?), et du linteau de la porte d'entrée en façade sud (figure 5b). Étant donné les difficultés d'accès à ce site, ces deux pièces sont sans doute des pièces de remploi sur place (ou provenant éventuellement des ruines situées immédiatement à l'aval). Les échantillons dont le dernier cerne est conservé - dont la majorité conserve encore l'écorce - indiquent un abattage en 1868 (figure 5e). Le bois final de ce cerne est quasi complet à complet, on peut donc en déduire un abattage entre la fin de l'été 1868 et la fin de l'hiver 1868/69.

En conclusion, l'analyse dendrochronologique indique que ce bâtiment était vraisemblablement présent dès le $\mathrm{XVII}^{\mathrm{e}}$ siècle, comme le suggère une pièce en remploi. Toute la charpente et le plancher ont, par la suite, fait l'objet d'une réfection au XIx ${ }^{e}$ siècle (ca. 1870) dans un chalet plus ancien. Cette hypothèse est renforcée par le fait que deux bâtiments sont déjà indiqués à cet emplacement sur la carte d'État Major, levée sur la zone en 1864 (Rühl, 2010). La principale période d'abattage déterminée ici est donc postérieure à la première implantation du chalet.

\section{Chalet au Covier (COV)}

Ce chalet est remarquable par la mise en ouvre de la maçonnerie, plus élaborée et soignée. L'échantillonnage a porté seulement sur quatre pièces de charpente en façade ouest (amont) (figure 6a/b). Deux sont des entraits (COV01 et COV02) et deux sont des pannes sablières superposées (COV03 et COV04). Des sondages ont également été effectués dans le linteau et le seuil de la porte de cette même façade, mais ceux-ci ont révélé trop peu de cernes pour envisager une datation. D'autres pièces de charpente auraient également pu être échantillonnées, notamment dans la partie où celle-ci est effondrée (figure 6b), mais l'accès à l'intérieur du bâtiment n'a pas été autorisé par le propriétaire (EDF) en raison du risque d'écroulement du toit et du plancher.

L'essence utilisée sur ce site est exclusivement l'épicéa. Le résultat des datations est présenté sur la figure 6c-e. Les séries corrèlent entre elles de façon significative (intercorrélation : 0,53). L'âge moyen des arbres lors de leur abattage était de $126 \pm 23$ ans $(\mathrm{n}=4)$. La datation des séries individuelles et de la moyenne de site a été faite sur les chronologies régionales suisses et autrichiennes avec de très bons résultats, confirmés par la chronologie locale du Bois de Besseil (Avrieux, Maurienne) (figure 6e; Le Roy et al., 2016). Ces 


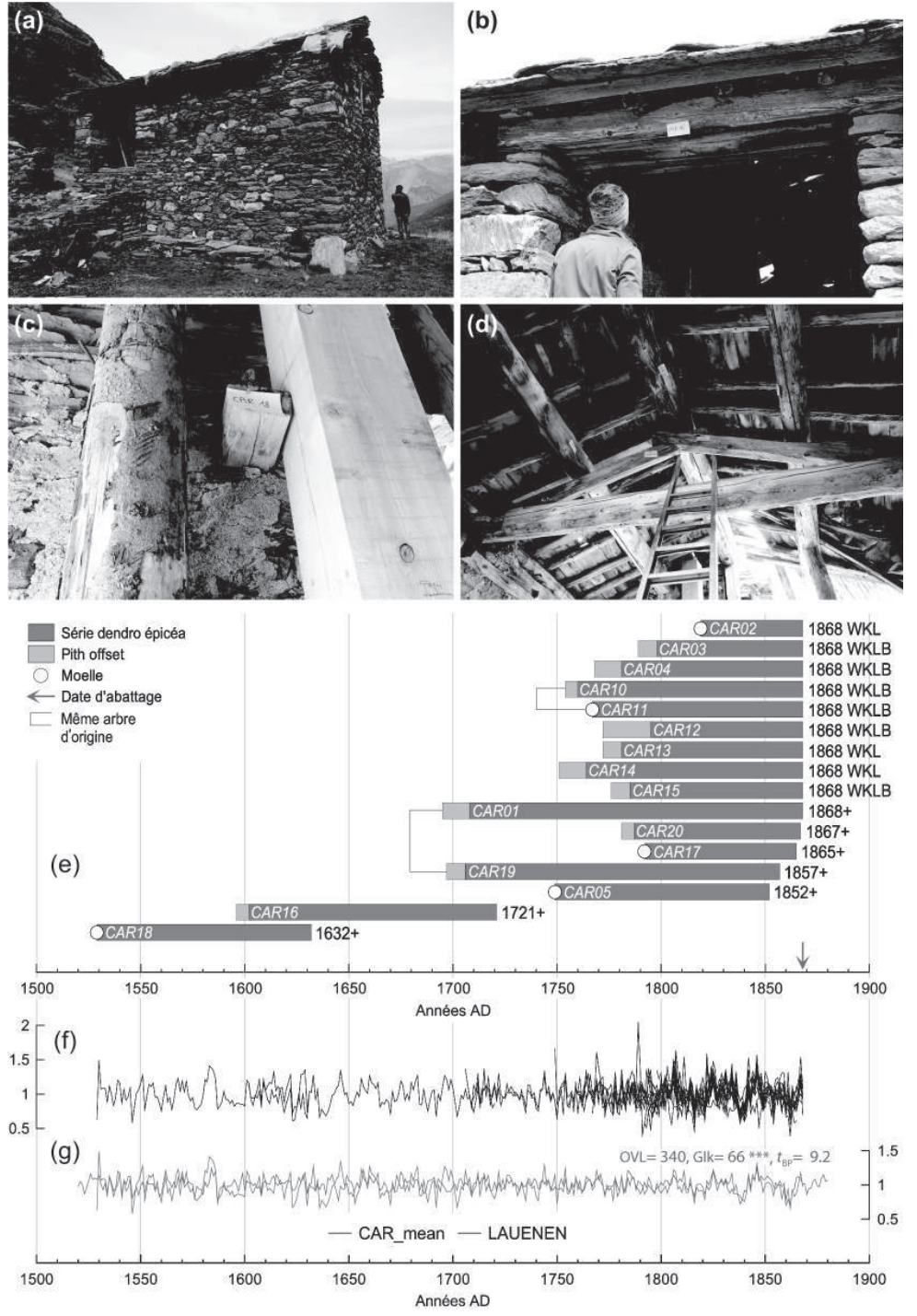

Figure 5 : (Voir planche couleur V) Chalet au Carroley (2122 m), parcelle 1718 .

(a) Vue d'ensemble de la façade sud (cliché: 15.09.15); (b) Linteau de la porte en façade sud (CAR16, datation: 1721+) ; (c) Pièce maçonnée dans le pignon ouest, vue de l'intérieur (CAR18, datation : 1632+) ; (d) Ferme et pannes échantillonnées (datation de l'ensemble : 1868). Les deux arbalétriers (CAR10 et 11) proviennent du même arbre; (e) Résultats des datations dendrochronologiques effectuées sur le chalet au Carroley. La nomenclature utilisée pour la présentation des dates d'abattage est détaillée dans le texte (Section 3.3); (f) Séries individuelles indicées à l'aide d'une moyenne mobile sur 11 ans; (g) Moyenne de site CAR en position de synchronisation sur la chronologie de référence d'épicéa LAUENEN (Canton de Berne, Suisse) et résultats des tests statistiques.

Figure 5: (See colour plate V) Chalet at Carroley (2 $122 \mathrm{~m}$ a.s.l.), parcel 1718.

(a) Overview of the southern facade (photograph: 15.09.15); (b) Lintel of the southern facade door (CAR16, dating: 1721+); (c) Masoned piece in the western gable wall, seen from inside (CAR18, dating: 1632+); (d) Sampled roof truss and beams (dating: 1868). Principal rafters (CAR 10 and 11) originate from the same tree; (e) Results of tree-ring dating performed on the chalet at Carroley. Nomenclature used for the presentation of felling dates is detailed in main text (Section 3.3); (f) Individual series indexed using a 11-year moving average; (g) CAR site mean series in crossdating position on the LAUENEN spruce reference chronology (Canton of Bern, Switzerland). Crossdating results are also shown. résultats indiquent que l'épicéa est datable sur des références distantes extra-régionales, et pas seulement sur des références locales.

Deux dates d'abattage proches mais distinctes ont été déterminées : à l'été 1863 (seulement quelques cellules de bois final sur ce cerne) pour les échantillons COV02 et COV03, et entre la fin de l'été 1864 et la fin de l'hiver 1864/65 (bois final complet) pour les échantillons COV01 et COV04 (figure 6c).

\section{Discussion}

Cette étude dendrochronologique exploratoire du bâti vernaculaire de la zone cœur du Parc National de la Vanoise a permis de dégager plusieurs grandes lignes :
1) Les dates d'abattage les plus anciennes identifiées ici sont relativement synchrones. Elles se situent dans la première moitié $\mathrm{du} \mathrm{Xv}^{\mathrm{e}}$ siècle sur plusieurs sites (CIV, POL, $\mathrm{CHA}$ ) (figure 7). En l'absence de prospection archéologique, elles datent une première phase de construction et donc la première occupation de ces sites. Sur certains bâtiments, ces bois les plus anciens sont faiblement représentés du fait de remaniements ultérieurs (CHA, POL), alors que sur d'autres ils sont encore nombreux (CIV). Le site CIV se singularise par l'ancienneté moyenne des bois et par la présence de l'arbre le plus ancien daté dans cette étude. Il est intéressant de noter que cette première date d'abattage au milieu du $\mathrm{XIV}^{\mathrm{e}}$ siècle intervient dans un hiatus dans les dates d'abattage de la vallée de la Clarée, $40 \mathrm{~km}$ au sud (figure 7; Édouard et Thomas, 2008; Édouard, 2010b). Elle est en revanche cohérente avec les plus anciennes dates d'abattage recensées 
Figure 6: (Voir planche couleur VI) Chalet au Covier (1300 m), parcelles 1635-36.

(a) Localisation des échantillons prélevés dans la façade ouest (cliché: 16.09.15) ; (b) Plan de la façade ouest (haut) et est (bas) (levés et dessin: C. Iancovescu); (c) Résultats des datations dendrochronologiques effectuées sur le chalet au Covier. La nomenclature utilisée pour la présentation des dates d'abattage est détaillée dans le texte (Section 3.3); (d) Séries individuelles indicées à l'aide d'une moyenne mobile sur 11 ans; (e) Moyenne de site COV en position de synchronisation sur la chronologie d'épicéa JURA-LUT-M (Jura suisse) et résultats des tests statistiques. Les résultats sur les chronologies d'épicéa LAUENEN (canton de Berne, Suisse) et BESS (Avrieux, Maurienne) sont également indiqués.

Figure 6: (See colour plate VI) Chalet at Covier (1300 $m$ a.s.l.), parcels 1635-36.

(a) Location of the samples taken in the western facade (photograph: 16.09.15); (b) Elevation sketch of the western facade (upper inset) and eastern facade (lower inset) of the chalet (survey and sketch: C. Iancovescu); (c) Results of tree-ring dating performed on the chalet at Covier. Nomenclature used for the presentation of felling dates is detailed in main text (Section 3.3); (d) Individual series indexed using a 11-year moving average; (e) COV site mean series in crossdating position
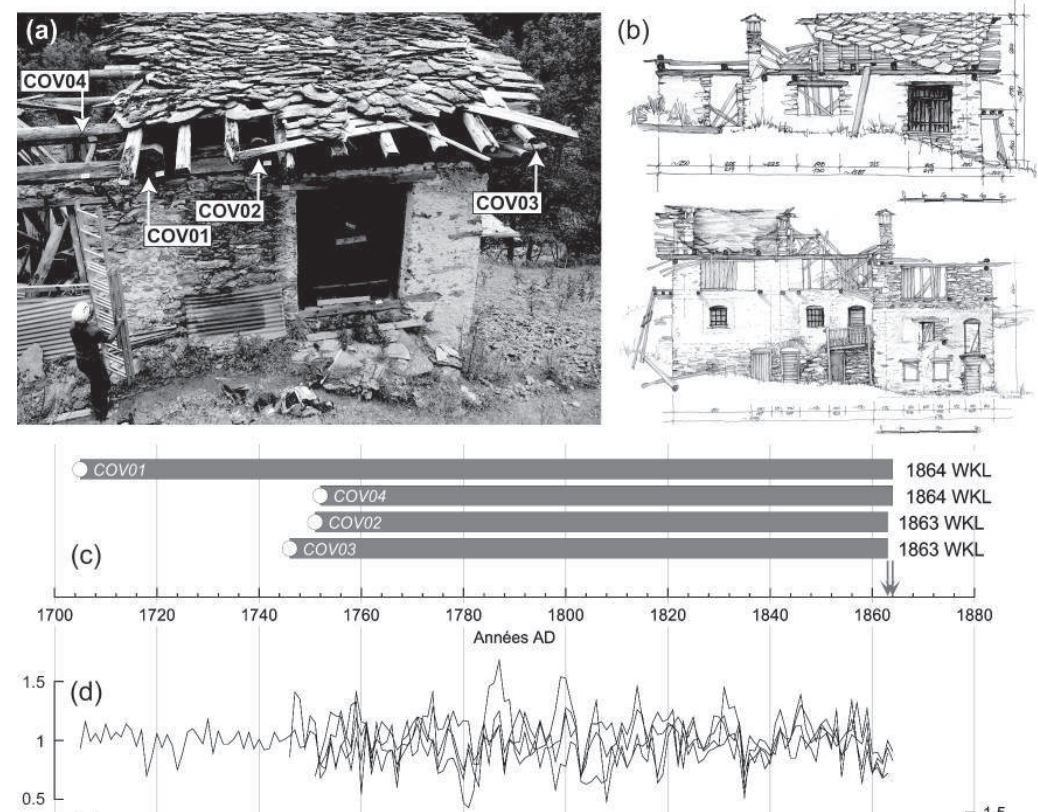

(e)

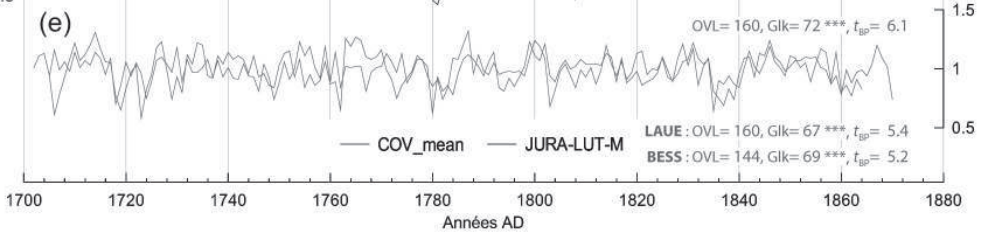
on the JURA-LUT-M spruce reference chronology (Swiss Jura). Crossdating results are shown on this reference and also on the LAUENEN spruce chronology (Canton of Bern, Switzerland) and the local BESS spruce chronology (Avrieux, Maurienne).

dans le Val d'Aoste (Remacle, 2014). Plus généralement, les premières dates d'abattage généralisées au $\mathrm{Xv}^{\mathrm{e}}$ siècle déterminées en Vanoise sont concordantes, à l'échelle alpine, avec d'autres études similaires sur le bâti d'altitude (e.g. Nicolussi et al., 2007; Édouard et Thomas, 2008; Édouard, 2010a; 2010b; Remacle, 2014).

2) Les interventions successives sur le bâti. On peut noter ainsi le remploi de grosses pièces de charpente dans un bâtiment construit/refait ultérieurement (e.g. les pannes faitières du XVII ${ }^{e}$ siècle sur les sites POL et CHA). À l'inverse, sur le site $\mathrm{CAR}$, le remploi concerne des pièces plus modestes, qui seraient potentiellement d'origine.

3) La période d'abattage déterminée pour les bois dont le dernier cerne formé est présent se place majoritairement entre la fin de l'été et la fin de l'hiver suivant (bois final formé entièrement ou quasi entièrement). Très peu d'échantillons ont formellement été identifiés comme ayant été abattus au printemps. Ces conclusions rejoignent celles de Klein et Grabner (2015) qui ont étudié des bâtiments de plus faible altitude en Autriche.

4) Lorsque le contexte le permet (c.-à-d. la présence d'un ou plusieurs chronogrammes et l'analyse d'échantillons pos- sédant le dernier cerne formé), le temps de stockage séparant l'abattage de la mise en œuvre des pièces de bois a été déterminé précisément. Un délai de deux ans a ainsi été mis en évidence sur les sites CIV (au XviI ${ }^{\mathrm{e}}$ siècle) et POL (au $\mathrm{XVIII}^{\mathrm{e}}$ siècle). Mais des délais plus longs ont aussi été observés (jusqu'à 10 ans à CIV au Xve siècle), ce qui est intéressant et nouveau sur les granges d'altitude. En effet, les pratiques en plaine ou dans les hautes vallées du Briançonnais vont plutôt dans le sens d'une mise en œuvre immédiate (Hoffsummer, 2009; Shindo, 2016). Sur le site CIV, très éloigné des forêts, l'explication réside sans doute dans un remploi rapide sur place de pièces d'un bâtiment détruit par une avalanche, ou bien de pièces initialement utilisées au village et remployées à l'alpage quelques années après.

5) Lorsque deux essences différentes sont mises en œuvre (e.g. POL, CHA), le mélèze paraît préférentiellement utilisé pour les éléments principaux de structure (pannes de charpente, faîtière) et l'épicéa pour des éléments d'importance secondaire (chevrons, solives, planches de volige). 


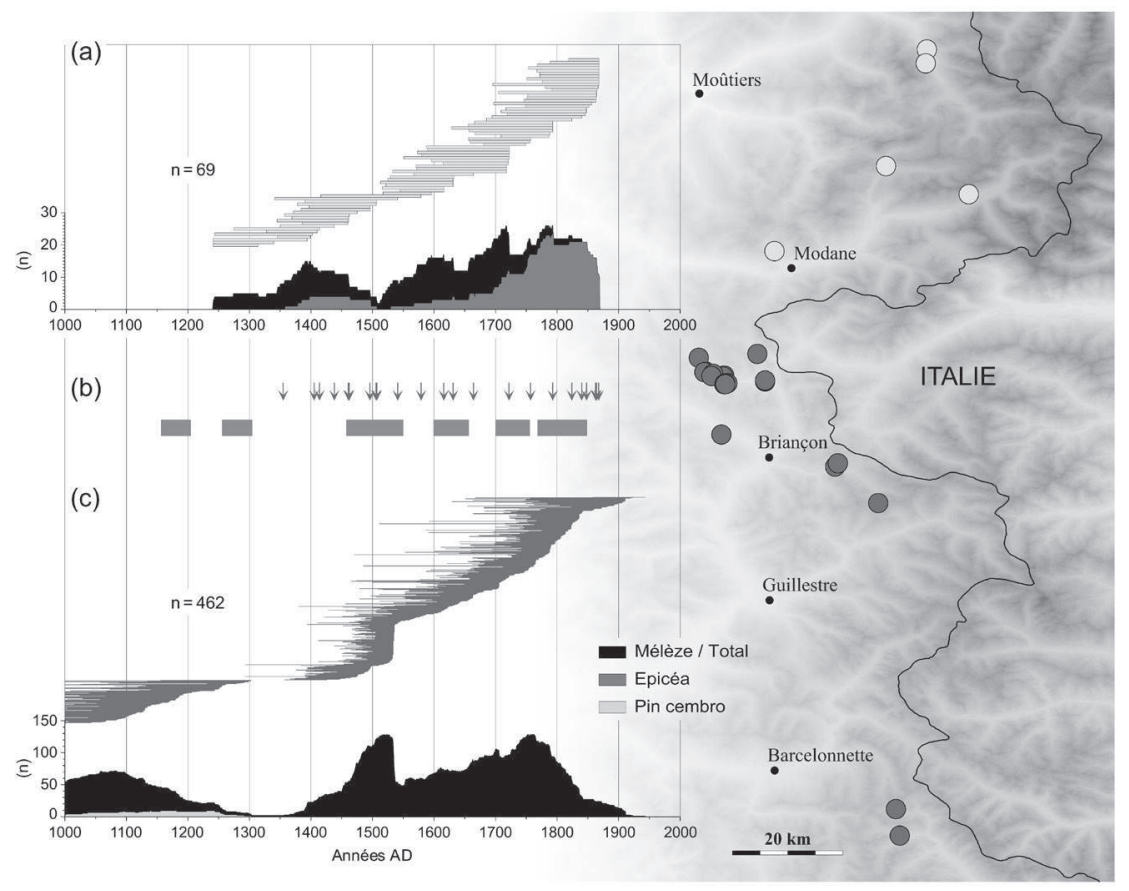

Figure 7 : (Voir planche couleur VII) Chalets d'alpage datés dans les Alpes françaises au cours du dernier millénaire.

(a) Séries dendrochronologiques des pièces de bois datées dans le cadre de cette étude, triées par date d'abattage (haut) et réplication des échantillons (bas). Les échantillons en place ne sont pas regroupés par arbre d'origine. L'essence est indiquée par la couleur; (b) Dates d'abattages déterminées en Vanoise (flèches; ce travail) et principales périodes d'abattage identifiées dans les Hautes-Alpes (rectangles; Shindo, 2016); (c) Séries dendrochronologiques des pièces de bois datées sur des chalets d'alpage dans les Hautes-Alpes, triées par date d'abattage (haut; données : J.-L. Édouard et L. Shindo) et réplication des échantillons (bas). L'essence est indiquée par la couleur. La localisation des bâtiments datés est indiquée sur la carte.

Figure 7: (See colour plate VII) Tree-ring dated mountain pasture buildings in the French Alps over the last millennium.

(a) Tree-ring series of the samples dated in this study sorted by felling date (yellow bars) and sample depth of the dated samples (lower panel). Series are not grouped by single original tree. Species is shown by different colours; (b) Felling dates determined in Vanoise (arrows; this study) and main felling periods identified in the southern French Alps (rectangles; Shindo, 2016); (c) Tree-ring series of the samples dated on mountain pasture buildings in the southern French Alps sorted by felling dates (red bars; data: J.-L. Edouard and L. Shindo) and sample depth of the dated samples (lower panel). Species is shown by different colours. Location of the dated buildings is shown on the basemap.

\section{Perspectives}

Cette étude préliminaire ouvre des pistes de travail à développer dans le cadre de programmes de plus grande ampleur. La première est d'ordre architectural : combiner l'approche dendrochronologique avec celle de l'archéologie du bâti afin de retracer plus finement les différentes phases d'intervention sur les bâtiments dans le temps et l'espace (déterminer précisément quelles parties ont été reprises et à quelle période). Le travail du bois (débits, outils, tracéologie, charpente) et son évolution au cours du temps pourrait également faire l'objet d'investigations.

La deuxième porte sur le volet historique. La combinaison avec les données historiques disponibles s'avère particulièrement nécessaire pour contextualiser les sites et mieux comprendre l'histoire des chalets, des alpages et de la pratique du pastoralisme. Les résultats dendrochronologiques peuvent orienter de nouvelles investigations des sources écrites (exploitations forestières, commerce du bois, etc.).

La dernière est d'ordre environnemental, le croisement des résultats avec des données issues de sources historiques et environnementales permettrait d'interpréter :
- les essences et les caractéristiques des arbres utilisés dans la construction, en relation avec la composition et la localisation des forêts actuelles (cartes de végétation, documents forestiers), ou passées (Mappe Sarde, carte d'état-major, etc.), pour préciser certains aspects du savoir-construire et identifier la provenance des bois;

- les patrons de croissance qui indiquent les types d'arbres utilisés (âge, croissance difficile ou non, perturbations, etc.) et donc renseignent sur les caractéristiques des forêts-ressource aux périodes d'abattage mises en évidence;

- les années avalancheuses. Malgré les précautions prises lors de la construction, des bâtiments d'alpage ont manifestement été soumis à cet aléa durant le Petit Âge Glaciaire (1250-1850 AD). L'identification de phases de réfection synchrones sur plusieurs sites (proches ou non) pourrait permettre d'appréhender cet aspect, en relation avec les chroniques historiques;

- les périodes de reconstruction et les hiatus dans les données dendrochronologiques peuvent résulter des fluctuations de l'activité agro-pastorale en relation avec les événements politiques et socio-économiques (migrations, guerres religieuses, révoltes, épidémies, incendies, changements de méthode d'exploitation/élevage). 
Bien sûr, la portée des conclusions est fonction de l'effectif du corpus. La prolongation de ce travail nécessiterait de dater un nombre critique de bâtiments (voire même de viser l'exhaustivité) au sein d'une zone géographique homogène et pertinente, afin de pouvoir tirer des conclusions robustes sur les dynamiques démographiques locales. Cela pourrait commencer à l'échelle d'un hameau et se poursuivre à l'échelle d'une commune ou d'une partie de vallée (e.g. Büntgen $e t$ al., 2005).

\section{Conclusion}

Cette étude dendrochronologique complète les informations architecturales de l'inventaire existant (Iancovescu, 2013; Bazan et Iancovescu, 2015). Les résultats mettent en lumière la richesse du bâti vernaculaire et renforcent sa valeur patrimoniale. Le bois est bien présent dans les constructions de la zone cœur pratiquement sans forêt, et ou la pierre semble omniprésente. Il permet de dater les premières constructions d'alpage à 700 ans (au moins) avant aujourd'hui, complétant ainsi les informations données par les dates gravées et renouvelant la lecture historique qui était faite de cet habitat.

\section{Remerciements}

Nous remercions les propriétaires Gilbert Rosaz, Serge Bonnevie et $M^{r}$ Dohy, ainsi que la mairie de Lanslevillard et EDF (notamment Bernard Tardy) pour l'aimable autorisation d'échantillonner les chalets. Kurt Nicolussi (Institut de Géographie, Université d'Innsbruck) nous a permis de tester plusieurs de nos séries sur des références non publiées. Nous remercions également chaleureusement Patrick Gassmann (Office du Patrimoine et de l'Archéologie de Neuchâtel) pour la communication de références d'épicéa suisses, ainsi que Claudine Remacle (architecte, Val d'Aoste) pour le partage de son expérience de l'architecture vernaculaire des Alpes occidentales. Enfin, merci aux deux relecteurs pour les remarques formulées sur la première version de ce manuscrit.

\section{Bibliographie}

Baillie M.G.L., Pilcher J.-R., 1973. A simple crossdating program for tree-ring research. Tree-Ring Bulletin, 33, p. 7-4.

Bazan M.-P., Iancovescu C., 2015. Trésors d'architecture au cour du Parc National de la Vanoise. Glénat, 160 p.

Brugière Y., Dubois H., Hoyrup E., Nicolas H., 2000. Guide de restauration des chalets d'alpage. Ed. Libris, 95 p.
Büntgen U., Bellwald I., Kalbermatten H., Schmidhalter M., Freund H., Frank D.C., Bellwald W., Neuwirth B., Nüsser M., EsPer J., 2006. 700 years of settlement and building history in the Lötschental/Switzerland. Erdkunde, 60/2, p. 96-112.

Corona C., Édouard J.-L., Guibal F., Guiot J., Bernard S., Thomas A., Denelle N., 2011. Long-term summer (AD 751-2008) temperature fluctuation in the French Alps based on tree-ring data. Boreas, 40, p. 351-366.

Dubois H., Hardy J.-P., Schwing K., 1994. Habitat traditionnel des vallées de Vanoise : Regards sur l'architecture. Rapport de la CAUE de la Savoie. Parc National de la Vanoise, Conseil général de la Savoie, $65 \mathrm{p}$.

EcksteIn D., BAUCH J., 1969. Beitrag zur Rationalisierung eines dendrochronologischen Verfahrens und zur Analyse seiner Aussagesicherheit. Forstwissenschaftliches Centralblatt, 88, p. 230-250.

Édouard J.-L., 2010a. Datation dendrochronologique du bâti traditionnel et occupation humaine dans les Alpes françaises du Sud au cours du dernier millénaire. In L. Astrade, C. Miramont (dir.). Panorama de la dendrochronologie en France, Digne-les-Bains, 8-10 octobre 2009, Collection EDYTEM, 11, Chambéry, p. 169-176.

ÉDOUARD J.-L., 2010b. Longue chronologie de cernes du mélèze et occupation humaine depuis plus de mille ans dans la vallée de la Clarée (Briançonnais, Alpes françaises). In X. Delestre, S. Tzortzis, J. Greck (dir.). Archéologie de la montagne européenne. Actes de la table ronde internationale de Gap, Musée - muséum départemental de Gap (Hautes-Alpes), 29-30 septembre 2008. Errance/Centre Camille-Julian, Bibliothèque d'Archéologie Méditerranéenne et Africaine, 4, p. 325-333.

Édouard J.-L., Thomas A., 2008. Cernes d'arbres et chronologie holocène dans les Alpes françaises. In M. Desmet, M. Magny, F. Mocci (dir.), Actes de la Table ronde JurAlp, Dynamique holocène de l'environnement dans le Jura et les Alpes : du climat à l'Homme, Aix en Provence, 15 et 16 novembre 2007, Collection EDYTEM 6, Chambéry, p. 179-190.

Gardent M., Rabatel A., Dedieu J.-P., Deline P., 2014. Multitemporal glacier inventory of the French Alps from the late 1960s to the late 2000s. Global and Planetary Change, 120 , p. 24-37.

Hermann M.-T., 1980. Architecture et vie traditionnelle en Savoie. Berger-Levrault, 229 p.

Hoffsummer P., 2009. Critical aspect of dendrochronology. In P. Hoffsummer (dir.). Roof frames from the $11^{\text {th }}$ to the $19^{\text {th }}$ century. Typology and development in northern France and Belgium. Analysis of CRMH documentation, Brepols, 376 p.

Iancovescu C., 2013. Inventaire du patrimoine bâti en Vanoise. Conseil scientifique du Parc National de la Vanoise, novembre 2013, Chambéry.

ArcheoSciences, revue d'archéométrie, 41(2), 2017, p. 7-22 
Kaennel M., Schweingruber F., 1995. Multilingual Glossary of Dendrochronology. Swiss Federal Institute for Forest, Snow and Landscape Research, Paul Haupt Publisher, 467 p.

Klein A., Grabner M., 2015. Analysis of Construction Timber in Rural Austria: Wooden Log Walls. International Journal of Architectural Heritage, 9, 5, p. 553-563.

Labbas V., 2016. Archéologie du bâti dans le massif du Mercantour du Moyen Âge à nous jours : Sources littéraires, matérielles et sources archéométriques. Thèse de doctorat en Archéologie, Université Aix-Marseille.

Le Roy M., 2012. Reconstitution des fluctuations glaciaires holocènes dans les Alpes occidentales, apports de la dendrochronologie et des datations par isotopes cosmogéniques produits in situ, Thèse de Doctorat en Géographie, Université de Savoie.

Le Roy M., Astrade L., Édouard J.-L., 2016. Connaissance de l'histoire du patrimoine bâti au cour du Parc National de la Vanoise, l'apport de la dendrochronologie. Rapport Laboratoire Edytem, Université Savoie Mont-Blanc CNRS, Centre Camille-Jullian, pour le Conseil Scientifique du Parc National de la Vanoise, 36 p.

[https://www.google.fr/url?sa=t\&rct=j\&q=\&esrc=s\&source=web\& $\mathrm{cd}=1 \& \mathrm{cad}=$ rja \&uact $=8 \&$ ved $=0$ ahUKEwju7KO6tbzPAhUM 2SwKHQPDCIoQFgghMAA\&url=http\%3A\%2F\%2Fwww. vanoise-parcnational.fr\%2Ffr\%2Fdownload $\% 2$ Ffile $\% 2$ Ffid $\%$ 2F909\&usg=AFQjCNFPTmFt3rHXIao_VAh2yPBKJz4lKA].

Mallé M.-P., 1999. L'habitat du nord des Hautes-Alpes. Patrimoine architectural et mobilier. Association pour le patrimoine de Provence et Société d'étude des Hautes-Alpes, 440 p.

Nicolussi K., Kaufmann M., Pindur P., 2007. Dendrochronologische Analyse der Bauentwicklung von Gebäuden der Waxeggalm im Zemmgrund, Zillertaler Alpen. In R. Luzian, P. Pindur (dir.), Prähistorische Lawinen - Nachweis und Analyse holozäner Lawinenereignisse in den Zillertaler Alpen, Österreich. BFW-Berichte 141 / Mitt. d.
Kommission für Quartärforschung d. Österr. Akademie der Wissenschaften, Wien, 16, p. 133-142.

ONde H., 1942. L'occupation humaine dans les grands massifs savoyards internes. Étude critique, historique et démographique. Arthaud, Grenoble, 359 p.

Pelletier O., 2003, Habiter la montagne : l'architecture rurale dans les Alpes occidentales. Bilan et perspectives de recherche. In G. Boetsch, W. Devriendt, A. Piguel (dir.). Permanences et changements dans les sociétés alpines : état des lieux et perspectives de recherche. Edisud, p. 197-214.

Remacle C., 2014. Architettura in legno in Valle d'Aosta XIV-XX secolo/Architecture de bois au Val d'Aoste XIV'-XX' siècle. Arti Grafiche Duc, Aoste, en collaboration avec l'architecte Danilo Marco, 416 p.

Raulin H., 1977a. L'architecture rurale française - Dauphiné. Berger Levrault, 277 p.

Raulin H., 1977b. L'architecture rurale française - Savoie. Berger Levrault, $230 \mathrm{p}$.

Rinn F., 2005. TSAP-Win Version 0.53. User reference. 110 p.

RüHL T., 2010. Apport des minutes de la carte d'État Major pour un inventaire des glaciers à la fin du Petit Âge Glaciaire. Mémoire de master 2 recherche, Université Joseph-Fourier, Grenoble.

Serre-Bachet F., 1978. The dendroclimatocological value of the European Larch (Larix decidua Mill.) in the French Maritime Alps. Tree-ring Bulletin, 38, p. 25-34.

SHindo L., 2016. Bois de construction et ressources forestières dans les Alpes du Sud au II millénaire: dendrochrono-écologie et archéologie. Thèse de doctorat en Archéologie, Université AixMarseille.

Siebenlist-Kerner V., 1984. Der Aufbau von Jahrringchronologien für Zirbelkiefer, Lärche, und Fichte eines alpinen Hochgebirgsstandortes. Dendrochronologia, 2, p. 9-29.

Tessier L., 1986. Chronologie de mélèzes des Alpes et Petit âge glaciaire. Dendrochronologia, 4, p. 97-113. 